acceptance after OGD [Condon 2008, Can J Gastro]. We assess tolerance and acceptability of MACE compared to OGD using the ECS.

Methods Patients referred for the endoscopic investigation of dyspepsia were recruited. Upper GI MACE examinations were performed followed by OGD. Questionnaires examined patients' tolerance to and acceptability of the endoscopies. Paired differences between median distress and ECS scores are reported $\left(\mathrm{p}<0.0001^{* * *}, \mathrm{p}<0.01^{* * *}\right)$.

Results Thirty eight (oral $\mathrm{n}=33$, transnasal $\mathrm{n}=5$ ) patients were recruited (median age 50, female 74\%, sedated in $36.4 \%$ of oral OGDs). All patients tolerated MACE adequately with minimal discomfort in $16 \%$. OGD was adequately tolerated in $63 \%$ with the remaining reporting moderate or severe discomfort. There was less distress ( 1 - 10: not at all - extremely) caused by: discomfort during ( 1 vs $5 * * *)$, discomfort after (1 vs $2 * * *)$ and pain during (1 vs $2 * * *)$ the procedures, gagging (1 vs $\left.6^{* * *}\right)$, choking ( 1 vs $\left.4.5^{* * *}\right)$, bloating ( 1 vs $2^{* *}$ ) and swallowing the capsule vs. insertion of the scope (1 vs $4 * * *)$ with MACE compared to OGD. The overall pre-procedure ECS score ( 28 vs 50 points $* * *)$ and post-procedure ECS score (13 vs 32 points***) was lower after MACE compared to OGD suggesting patients are more accepting of MACE before and after the procedure compared to OGD. There was a reduction in pre-procedure ECS after both MACE (median difference $-15 * *)$ and OGD $(-18 * *)$ suggesting procedures are not as distressing as initially anticipated, however $32 \%$ of patients report a higher ECS after gastroscopy whereas all patients reported a lower ECS after MACE. Patient preference towards MACE or OGD illustrated in table 1 below.

\begin{tabular}{|c|c|c|c|}
\hline & MACE & OGD & \\
\hline Patient preference & Yes, n (\%) & & $\mathrm{p}$ \\
\hline $\begin{array}{l}\text { Undergo the same test under the same } \\
\text { medical circumstances? }\end{array}$ & $38(100)$ & $30(79.0)$ & $* * *$ \\
\hline $\begin{array}{l}\text { Advise a friend to undergo the same test } \\
\text { given the same medical circumstances }\end{array}$ & $38(100)$ & $27(71.0)$ & $* * *$ \\
\hline $\begin{array}{l}\text { As a screening for cancer in } 1 \text { to } 2 \text { years } \\
\text { time? }\end{array}$ & $38(100)$ & $30(79.0)$ & $* * *$ \\
\hline $\begin{array}{l}\text { If the chance of requiring biopsy after } \\
\text { MACE was: }\end{array}$ & MACE first & OGD first & \\
\hline 1 in 20 & $10(91)$ & $1(9)$ & \\
\hline 1 in 10 & $10(91)$ & $1(9)$ & \\
\hline 1 in 5 & $9(82)$ & $2(18)$ & \\
\hline 1 in 4 & $8(73)$ & $3(27)$ & \\
\hline 1 in 2 & $8(73)$ & $3(27)$ & \\
\hline
\end{tabular}

Conclusions Patients better tolerate, are more accepting of and prefer MACE over OGD. Where biopsies are required, patients would still prefer capsule endoscopy initially. Cost effectiveness of this approach should be examined

\section{PTU-120 ROBOT CONTROLLED MAGNET-ASSISTED CAPSULE GASTROSCOPY PROVIDES NON-INFERIOR VIEWS TO CONVENTIONAL GASTROSCOPY: A PILOT STUDY}

David Tai* ${ }^{*}$ Hey Long Ching, Mark McAlindon. Sheffield Teaching Hospitals, Sheffield, UK

10.1136/gutjn--2019-BSGAbstracts.109
Introduction Oesophagogastro duodenoscopy (OGD) is undertaken by $1 \%$ of UK population per year but is often poorly tolerated. Capsule endoscopy is well tolerated. Robot controlled magnet assisted capsule endoscopy (MACE) allows control of a capsule endoscope within a pool of swallowed water resulting in a non-invasive endoscopic examination of the upper GI tract. We examine whether there is a difference in the endoscopic views between MACE and OGD.

Methods Patients referred for the endoscopic investigation of dyspepsia were recruited. Upper GI MACE examinations were performed followed by OGD. Endoscopic views of upper GI landmarks on MACE and video recordings of tandem OGDs are graded ( 0 - no views, 1- Poor: $>75 \%$ obscured, 2- Suboptimal: $>50 \%$ obscured, 3- Reasonable: $<50 \%$ obscured, 4Good: $<25 \%$ obscured, 5-Excellent: complete view). Obscuration at MACE defined as debris, bubbles, poor image clarity/ illumination, and at OGD as lack of insufflation, patient retching, mucus or debris. Clarity and distension of stomach is graded (1- poor, 2-reasonable, 3- good). Non-parametric paired differences between MACE and OGD were examined $\left(\mathrm{p}<0.005^{* *}, \mathrm{p}<0.05 \%\right)$.

Results Sixteen (peroral $\mathrm{n}=15$, transnasal $\mathrm{n}=2$ ) patients (median age of 49, female 76\%) were included. Mean examination time for MACE was longer at 46.4 minutes compared to conventional gastroscopy of 4.7 minutes $(\mathrm{p}<0.0001)$. No GOJ views were seen on MACE in $38 \%$ $(6 / 17)$. The capsule was steered into D1 in $12 \%(2 / 17)$ with views of duodenum in $76 \%(13 / 17)$. There were no differences in the reported clarity (2.5 vs 2$)$ and distension (3 vs 2) of stomach between MACE and flexible gastroscopy $(\mathrm{p}<0.05)$. Differences in median endoscopic view scores are show below.

Abstract PTU-120 Table 1 Paired endoscopic view comparisons of MACE and fibre optic gastroscopy

\begin{tabular}{|c|c|c|c|}
\hline Landmark & MACE & OGD & $p$ \\
\hline & Median (IQR) & & \\
\hline Oesophagus & $4.5(2)$ & $5(0)$ & * \\
\hline GOJ & $3(5)$ & $5(1)$ & ** \\
\hline \multirow[t]{2}{*}{ Cardia } & 5 & 4 & \\
\hline & $(0.75)$ & $(1.75)$ & \\
\hline \multirow[t]{2}{*}{ Fundus } & $5(0)$ & 4 & * \\
\hline & & $(2.75)$ & \\
\hline \multirow[t]{2}{*}{ Greater Curve } & 5 & $3.5(1)$ & ** \\
\hline & $(0.75)$ & & \\
\hline Lesser Curve & $5(0)$ & $4(2)$ & * \\
\hline \multirow[t]{2}{*}{ Anterior Body } & 5 & $4(1)$ & ** \\
\hline & $(0.75)$ & & \\
\hline Posterior & $5(1)$ & 4 & ** \\
\hline Body & & $(1.75)$ & \\
\hline Antrum & $5(0)$ & $5(0)$ & \\
\hline D1 & $3(2)$ & $5(0)$ & ** \\
\hline D2 & $4(1)$ & $5(0)$ & \\
\hline
\end{tabular}

Conclusions Unblinded comparisons suggest gastric landmark views are excellent and compared to swifter gastroscopy studies, may better visualise the body and fundus of the stomach. On the other hand, oesophageal and D1 views are superior with OGD and active transpyloric passage into the duodenum using the magnetic fields was limited. This endoscopic view score needs validation by blinded independent parties. Further 
work is required to develop oesophageal examination techniques using MACE.

\section{PTU-121 THE VALUE OF ENDOSCOPIC BIOPSIES IN ROUTINE UPPER GASTROINTESTINAL ENDOSCOPY}

David Tai*, Mustafa Jalal, Gloria Tun, Stefania Chetcuti-Zammit, Mark McAlindon. Sheffield Teaching Hospitals, Sheffield, UK

\subsection{6/gutjnl-2019-BSGAbstracts.110}

Introduction The BSG and AUGIS quality standards in upper gastrointestinal endoscopy recommend taking biopsies for histological examination to diagnose inflammatory, pre- or malignant lesions which may or may not be visible to the naked eye [Beg, Gut 2018]. This may increase the duration and cost of the procedure and potentially reduce the focus on mucosal inspection. This study aimed to investigate the diagnostic value (changes in diagnosis and contribution to management) added by histology and its cost.

Methods Patients undergoing consecutive diagnostic OGDs performed by four fellows were recruited. Incomplete, surveillance or therapeutic procedures, procedures for dysphagia and those arranged in order to obtain histology (eg. positive coeliac serology) were excluded. Biopsies were performed according to BSG quality standards [Beg, Gut 2018]. The utility of biopsies (changes in diagnosis and contribution to management) were examined from endoscopic and histological findings.

Results Of 509 patients examined, 314 were included. OGD was performed for dyspepsia (66\%), anaemia (26\%), weight loss (16\%), diarrhoea (2\%) and vomiting (1\%). Biopsies occurred in $86 \%$ of patients with an mean of 1.5 samples per patient biopsied. Biopsy sampling provided additional pathological information not evident at endoscopy in $28 \%$ of cases. However, if benign pathologies are excluded (mostly chemical or Helicobacter gastritis or duodenitis), this falls to $6.7 \%$ $(n=18$ : dysplasia $n=3$, adenocarcinoma $n=1$, gastric intestinal metaplasia (GIM) $n=7$, villous atrophy (VA) $n=2$, Barretts $\mathrm{n}=5$ ). In total, management change occurred in $19 \%$ consequent on histological findings, but this falls to $12 \% \quad(n=34$ : adenocarcinoma $\mathrm{n}=2$, dysplasia $\mathrm{n}=3$, GIM, $\mathrm{n}=10$, Barretts $\mathrm{n}=15$, VA $\mathrm{n}=3$, peptic stricture $\mathrm{n}=1$ ) where Helicobacter pylori (which can be detected by other non-invasive methods) eradication is excluded.

Fifty seven focal lesions were biopsied where dysplasia or cancer was diagnosed in 7\% (1/14 oesophageal, 2/35 gastric and $1 / 8$ duodenal). Barretts, GIM and VA were found more commonly in abnormal compared to normal mucosa (See table 1). The overall cost of tissue sampling approximates to $£ 17,100$ ( $£ 82$ per person). Routine biopsies of patients with only normal mucosa costed over $£ 3,500$.

Abstract PTU-121 Table 1 Diagnostic yield for premalignant conditions in abnormal vs normal mucosa

\begin{tabular}{llll}
\hline & Abnormal & Normal & $\mathrm{p}$ \\
\hline Barrett's & $\%(\mathrm{n})$ & & \\
GIM & $58 \%(14)$ & $0 \%(0)$ & 0.02 \\
Villous atrophy & $81 \%(9)$ & $18 \%(2)$ & 0.05 \\
\hline
\end{tabular}

Conclusions Although 86\% undergoing OGD have biopsies taken, contributing to aetiopathological understanding in $28 \%$, only a small minority $(6.7 \%)$ have pre-malignant or malignant pathologies and result in a change in management in only $12 \%$. Non-targeted biopsies are of limited value and costly when sampled from normal mucosa.

\section{PTU-122 A PILOT DATA IN UNDERSTANDING THE CAUSES \& TEMPORAL RELATIONSHIP OF ELECTIVE ENDOSCOPY UNPLANNED ADMISSIONS WITHIN 8DAYS}

Bee Theng Tan*, Irfan Amin. Peterborough City Hospital, Peterborough, UK

\subsection{6/gutjnl-2019-BSGAbstracts.111}

Introduction We aim to understand the causes and temporal relationship of unplanned admissions within 8 days of elective endoscopy as per JAG recommendation.

Background We retrospectively examined all unplanned admissions within 8 days of elective endoscopy from Jan-June 2018. Demographic and co-morbidity data as defined by Charlson Comorbidity Index (CCI) and causes of admissions were collected.

Unplanned admissions were divided into endoscopic related and non-endoscopic related causes. The former was further divided into direct (perforation, infection, bleeding, and postpolypectomy syndrome) and indirect (sedation-related, displaced NG/NJ tube, or abdominal pain) complications.

Admissions were then classified as early (Day 0-2) or late (Day 3-8). Data was collected using Microsoft Excel and analyzed for any association between endoscopic complications with admission time.

Results Of the 5482 elective endoscopy patients, 65(1.18\%) had unplanned admissions within 8 days. The majority 62/65 (95\%) had 1 or more co-morbidities (CCI score $\geq 1$ ). High CCI scores may thus be a risk factor for unplanned admissions. Of all endoscopic procedures, ERCP had the highest admissions rate. 12 of $164(7.3 \%)$ patients undergoing elective ERCP had unplanned admissions. Colonoscopy and sigmoidoscopy had the lowest unplanned admission rate at $12 / 2038$ $(0.5 \%)$ and $4 / 788(0.5 \%)$ respectively.

Of the 65 unplanned admissions, 26/65(40\%) were admitted within the first 72 hours and 39/65(60\%) thereafter. Most early admissions $(14 / 26=54 \%)$ were related to endoscopic complications while the remaining were due to a non-endoscopic cause $(12 / 26=46 \%)$.

In contrast, the majority of late admissions were related to non-endoscopic causes $(31 / 39=80 \%)$. Endoscopy related complications in this timeframe were minimal $(8 / 39=20 \%)$.

Overall, ERCP has the highest risk of direct $(8 / 164=4.8 \%)$ and indirect complications $(2 / 164=1.2 \%)$ amongst the unplanned admissions. In addition, ERCP carries a significantly higher risk of direct than indirect complications $(p=0.0361)$. This may therefore explain the highest unplanned admissions rate found in ERCP compared to other endoscopic procedures.

Summary Our study provides crude data analyzing the risk of unplanned admissions related to elective endoscopic procedures in a busy DGH endoscopy unit. We aimed to understand if there was any temporal relationship in unplanned admissions post-elective endoscopy. Subsequent data demonstrated majority of direct complications of endoscopy were admitted within the first 72 hours with ERCP carrying the 